\title{
Note on the Linear Matrix Equation.
}

By J. H. Maclagan-W edderburn, M.A.

The general linear matrix equation has been investigated by Sylvester ${ }^{*}$ in a series of interesting papers published in the Comptes Rendus. His method, however, does not show that the solution can be exhibited as an analytical function of the coefficients. The object of this note is to supply such a solution.

The term, analytic function of the matrices $\phi_{1}, \ldots \phi_{n}$, is used in this paper to denote any function of $\phi_{1}, \ldots \phi_{n}$, which can be developed in a convergent series involving only powers of the variables and constants, i.e., a series of the form

$$
f(\phi)=\theta_{1}+\Sigma \theta_{2} \phi \theta_{3}+\Sigma \theta_{4} \phi \theta_{5} \phi \theta_{6}+\ldots
$$

where the $\theta$ 's are matrices independent of $\phi$.

The simplest case of the linear matrix equation is

$$
x-\phi \times \psi=\theta
$$

This gives

$$
\chi=\theta+\phi \chi \psi
$$

which, when substituted in the second term of the left-hand side of (1), leads to

$$
\chi-\phi^{2} \chi \psi^{2}=\theta+\phi \theta \psi
$$

and, on repeating the process $n$ times,

$$
\begin{aligned}
\chi-\phi^{n+1} \chi \psi^{n+1} & =\theta+\phi \theta \psi+\phi^{2} \theta \psi^{2}+\ldots+\phi^{n} \theta \psi^{n} \\
& =\theta_{n} \quad-\quad-\quad-\quad-
\end{aligned}
$$

From this it is evident that the series $\mathrm{L}_{n=\infty} \theta_{n}$, when convergent, is a solution of (1).

* Comptes Rendus, I.C., 1884, p. 67, 115, 117, 527. 
The general linear equation

when arranged in the form

$$
\Sigma \phi \chi \psi=\theta,
$$

$$
\chi-\Sigma \phi_{1} \chi \psi_{1}=\theta,
$$

can be treated in the same way, the solution being

$$
\chi=\theta+\Sigma \phi_{s} \theta \psi_{s}+\ldots \ldots+\Sigma \phi_{s_{1}} \phi_{s_{2}} \ldots \phi_{s_{n}} \theta \psi_{s_{n}} \ldots \psi_{s_{2}} \psi_{s_{1}}+\ldots
$$

This can be put in the simple form

$$
\chi=\theta+\Phi \theta+\Phi^{2} \theta+\ldots \quad-\quad-\quad-\quad \text { - }
$$

where $\Phi$ denotes the operation $* \Sigma \phi_{s}() \psi$.

i.e., $\Phi$ is a matrix of order $n^{2}$ where $n$ is the order of the matrices $\theta, \chi$, etc.

The series

evidently converges if

$$
1+\Phi+\Phi^{2}+\ldots
$$

$$
1+g+g^{2}+\ldots \ldots
$$

converges for all the roots of $\Phi$. (See E. Weyr, Bull. des Se. Math., 11 (1887), p. 205.) It is obvious that, by manipulating the original equation, we can in general secure the convergence of these series.

This method of solution does not depend on the nature of the symbols used, and can be applied in a variety of problems.

We may use it, for instance, to solve the equation

$$
\frac{\hat{\partial} \chi}{\partial t_{1}}+\Sigma \phi_{r} \frac{\partial \chi}{\partial t_{r}} \psi_{r}=\frac{\partial \chi}{\partial t_{1}}-U_{\chi}=\theta
$$

The inverse of $\frac{\hat{o}}{\hat{\partial} t_{1}}$ is $\int_{a_{1}}^{t} d t_{1}, \alpha_{1}$ being an arbitrary function of $t_{2} \ldots t_{u}$, and the solution of (4) is accordingly

$$
x=\int_{a_{1}}^{t_{1}} d t_{1} \theta+\int_{a_{2}}^{t_{1}} d t_{1} \mathrm{U} \int_{a_{1}}^{t_{1}} d t_{2} \theta+\int_{a_{3}}^{t_{1}} d t_{1} \mathrm{U} \int_{a_{1}}^{t_{1}} d t_{1} \mathrm{U} \int_{a_{2}}^{t_{1}} d t_{1} \theta+\ldots \ldots
$$

* Sylvester Comptes Rendus, 1884, p. 117. 
This method is capable of considerable extension, e.g., the solution of

$$
\begin{gathered}
\frac{\partial^{2} f}{\partial x^{2}}+\xi(x, y, z) \frac{\partial^{2} f}{\partial y^{2}}+\eta(x, y, z) \frac{\partial^{2} f}{\partial z^{2}} \equiv \frac{\partial^{2} f}{\partial x^{2}}+\mathrm{U} f=0 \\
\text { is * } f=\left(1-\int_{x_{2}}^{x} d x \int_{x_{1}}^{x} d x \mathrm{U}+\int_{x_{4}}^{x} d x \int_{x_{3}}^{x} d x \mathrm{U} \int_{x_{2}}^{x} d x \int_{x_{1}}^{x} d x \mathrm{U} \ldots \ldots\right) g(y, z) \\
+\left(\left(x-x_{0}\right)-\int_{x_{2}}^{x} d x \int_{x_{1}}^{x} d x \mathrm{U}\left(x-x_{0}\right) \ldots \ldots \ldots \ldots \ldots \ldots \ldots \ldots . . . \ldots\right) h(y, z)
\end{gathered}
$$

where $x_{0}, x_{1}, \ldots g(y, z)$ and $h(y, z)$ are arbitrary functions of $y$ and $z$. If all the lower limits of integration are equal to $x_{0}$, the solution is such that, when $x=x_{0}$, then $f=g(y, z)$ and $\frac{\partial f}{\partial x}=h(y, z)$. The solution therefore satisfies Cauchy's initial conditions. The convergency of such series is often easily investigated. They also give a variety of ways of expanding functions in series whose convergence is readily tested. All the quantities used above may be matrices except the independent variables and the limits of integration.

The following method of solving the equation

$$
\phi \chi-\chi \psi=\theta
$$

is a direct generalisation of the method which Hamilton used for the corresponding equation in quaternions. Its chief interest lies in showing the connection between the methods of Hamilton and Sylvester. Taber $\dagger$ has defined a series of matrices denoted by $\mathrm{K} \phi, \mathrm{K}^{2} \phi \ldots \mathrm{K}^{n-1} \phi$ which are obtained from $\phi$ by $1,2, \ldots n-1$ cyclical permutations of its roots, $n$ being the order of $\phi$. He has also shown that the following relations subsist between them :

* Sturm (Cours d'Analyse II., p. 146, \$614) has obtained the same series by a somewhat similar method for the equation $\frac{d^{2} y}{d x^{2}}+I y=0$. He, however, makes all his limits of integration the same. This series has also been obtained by different methods by other authors, e.g., Caqué, Liouville's Journal, Ser. II., Vol. IX., 1864, and Fuch's Annali di Math., Ser. II., Vol. 4, p. 46. See also Trans. Roy. Soc. Edin., XL., 1903. This method of solution can evidently be extended to equations of any order.

† Amer. Joumi. of Math., Vol. XII. (1890), p. 388. 


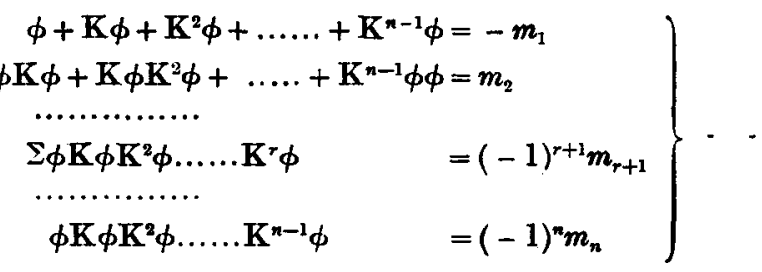

where $m_{1}, m_{2}, \ldots$ are the coefficients in the characteristic equation of $\phi$, viz.,

$$
\phi^{n}+m_{1} \phi^{n-1}+\ldots \ldots+m_{n}=0
$$

We can now derive from (5) a series of equations of the form

$$
\begin{array}{r}
\mathrm{K}^{r_{1}} \phi \mathrm{K}^{r_{2}} \phi \ldots \mathrm{K}^{r_{n-1}} \phi \phi \chi \psi^{s}-\mathbf{K}^{r_{1}} \phi \ldots \mathbf{K}^{r_{n-1}} \phi \chi \psi^{s+1} \\
=\mathrm{K}^{r_{1}} \phi \ldots \mathrm{K}^{r_{n-1}} \phi \theta \psi^{s},
\end{array}
$$

the $r$ 's being given all values from 0 to $n-1$ with the following restrictions: (1) expressions differing only in the order of their terms are to be regarded as identical ; $(2)$ in one expression none of the $r$ 's are to be equal ; (3) $s=n-1-r_{1}-r_{2} \ldots-r_{n-1} \nless 0$.

Adding all these equations together we get the following result :

$$
\begin{aligned}
& \mathrm{K}^{n-1} \phi \mathrm{K}^{n-2} \phi \ldots \mathrm{K} \phi \phi X-\left(\mathrm{SK}^{n-2} \phi \mathrm{K}^{n-1} \phi \ldots \mathrm{K} \phi \phi\right) \chi \psi+\ldots \ldots \\
& \begin{aligned}
\pm\left(\phi+\mathrm{K} \phi+\mathrm{K}^{2} \phi+\ldots+\mathrm{K}^{n-1} \phi\right)+(-1)^{n} \chi \psi^{n}=\mathrm{K}^{n-1} \phi \mathrm{K}^{n-2} \phi \ldots \mathrm{K} \phi \theta+\ldots \\
=\theta
\end{aligned} \\
& \text { i.e., } \quad x\left(m_{n}+m_{n-1} \psi+\ldots+\psi^{n}\right)=-\theta . \quad \\
& \therefore \quad x=-\theta\left(m_{n}+m_{n-1} \psi+\ldots+\psi^{n}\right)^{-1} .
\end{aligned}
$$

This is equivalent to Sylvester's form in which $\theta$ is obtained as

$$
\begin{aligned}
& \left(\phi^{n-1} \theta+\phi^{n-2} \theta \psi+\ldots+\theta \psi^{n-1}\right) \\
+ & m_{1}\left(\phi^{n-2} \theta+\ldots+\theta \psi^{n-2}\right) \\
+ & \ldots \ldots \ldots \ldots \ldots . . \\
+ & m_{n-1}(\phi \theta+\theta \psi) .
\end{aligned}
$$

A curious consequence of this result is that if $\psi$ has the same characteristic equation as $\phi$, then

must satisfy the linear equation

$$
\theta=\phi \chi-\chi \psi
$$

$$
\theta=0
$$


If $\phi=\psi$ we can also find as follows a set of scalar conditions satisfied by the coefficients of $\theta$.

Substituting $\phi+k \theta$ for $\phi$ in (7) and equating the coefficient of $k$ to zero, we get the following equation *

$$
\Theta+a_{1} \phi^{n-1}+a_{2} \phi^{n-2}+\ldots+a_{n}=0,
$$

where $a_{1}, \ldots a_{n}$ are invariants of the two matrices $\phi$ and $\theta$; therefore, as $\theta=0$, we must also have

$$
a_{1}=0, a_{2}=0, \ldots \ldots a_{n}=0 .
$$

Similar conclusions can be drawn with regard to the invariants of $x$ and $\theta$.

* Cf. Sylvester, Amer. Journ. of Math., VI., 1884, p. 279. 